Journal of Accident and Emergency Medicine 1995 12, 134-137

\title{
Chainsaw penetrating neck injury
}

\author{
A.F.T. BROWN \\ Department of Emergency Medicine, Royal Brisbane Hospital, Brisbane, Queensland, Australia
}

\section{SUM MARY}

A case of chainsaw injury to the neck is described. Previous reports in the English language are exceedingly rare. A brief discussion of safety features on chain saws is followed by a review of selective vs. mandatory surgical exploration in penetrating neck trauma, including the role of ancillary diagnostic tests.

Key words: neck injuries, penetrating neck injuries, traumatology, wounds and injuries

\section{INTRODUCTION}

Penetrating neck trauma is uncommon, with most injuries caused by stab wounds, gunshot wounds or shotgun wounds. ${ }^{1}$ Overall mortality rates range from $3 \%$ to $6 \%$, caused by haemorrhagic shock, asphyxiation, cerebral infarction, spinal cord injury, air embolism or sepsis. ${ }^{2}$ The anatomy of the neck is divided into three horizontal zones that relate to both the pathophysiology and management. Zone I extends from the root of the neck and upper mediastinum to below the cricoid cartilage. Zone II is the neck proper from the level of the cricoid cartilage to the angle of the mandible, and Zone III extends from the base of the skull cephalad to the angle of the mandible. ${ }^{3}$ Stab wounds and Zone II injuries have lower morbidity and mortality rates than missile wounds and Zone I or III injuries. ${ }^{4}$ Open chainsaw injury to the neck is exceedingly rare, with only sporadic cases reported previously in the English language. ${ }^{5} \mathrm{~A}$ recent case is reported and safety features designed to minimize injury during

Correspondence: A.F.T. Brown, Staff Specialist, Department of Emergency Medicine, Royal Brisbane Hospital, Brisbane, Queensland 4029, Australia chainsaw use are reviewed. Selective vs. mandatory surgical exploration and the role of ancillary diagnostic tests in penetrating neck trauma are then discussed.

\section{CASE REPORT}

A Medivac Team from the Royal Brisbane Hospital consisting of the author and a critical care nurse was recruited to assist a 32-year-old man trapped 20 feet up a tree. He had sustained a major laceration to the left side of his neck from a chainsaw which had 'kicked back' after hitting a knot in a branch. The Fire Brigade finally managed to free the patient $\mathbf{2 1}$ min after the injury, just as the Medivac Team arrived. Considerable blood loss was noted on the patient's clothing, on the tree trunk and on the ground below. On examination, the patient had an unobstructed airway, midline trachea, and an increased respiratory rate of 20 , but with equal, bilateral air entry. His pulse was 60 and his blood pressure was $115 / 60 \mathrm{mmHg}$. There was a large, ragged laceration to the left neck, with continuous non-arterial bleeding. The patient was fully conscious and orientated. The wound was immediately covered with a dressing, applying direct pressure, and two large-bore intravenous cannulae were inserted to initiate modest fluid replacement of $250 \mathrm{~mL}$ Haemaccel and $200 \mathrm{~mL}$ normal saline.

On arrival in the Emergency Department at the Royal Brisbane Hospital 43 min following injury, the patient was placed in slight Trendelenburg position on the Resuscitation Room trolley. Vital signs remained normal, although moderate pressure was required to prevent bleeding from the neck wound that extended from the submental region $2 \mathrm{~cm}$ below the mandible, to posterior and inferior to the left ear (see Fig. 1). The sternomastoid muscle was clearly divided, as was the external jugular vein. Immediate surgical exploration in theatre was arranged by the on-call general surgeon, whilst the patient was catheterized and given intravenous cefotaxime $1 \mathrm{~g}$, fentanyl and antitetanus prophylaxis. A chest radiograph was normal.

Surgical exploration revealed that the sternomastoid and strap muscles had been severed, and the external and internal jugular veins divided. The hyoid bone was fractured on the left, and the thyroid cartilage split without involvement 


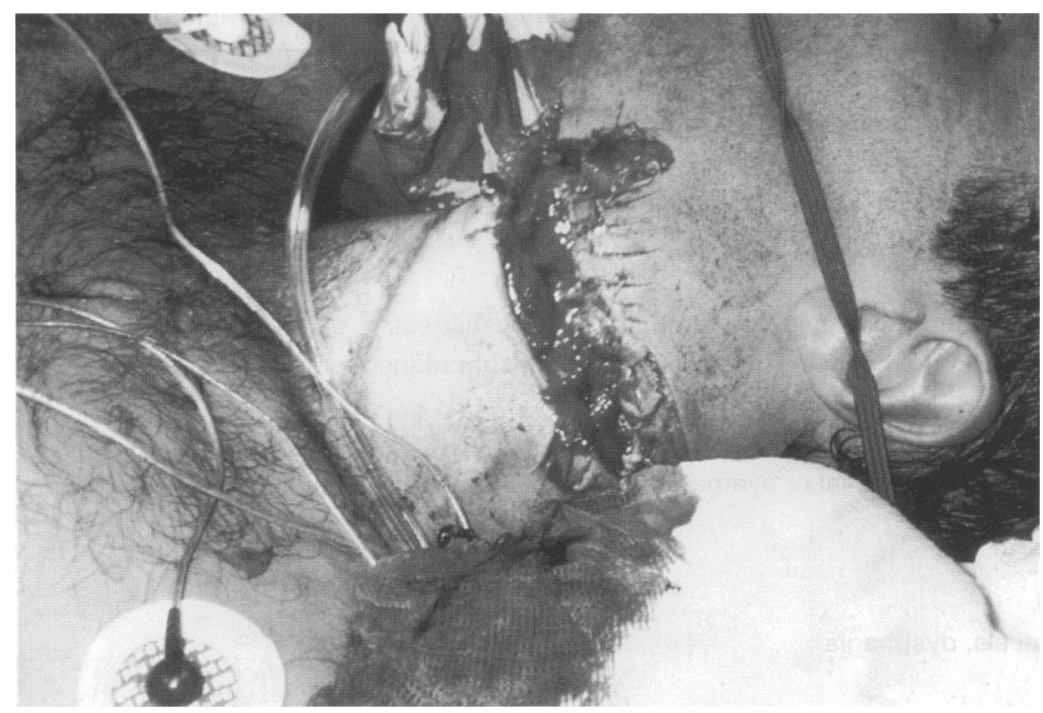

Fig. 1. Chainsaw injury to left neck.

of the larynx. The internal carotid artery, accessory nerve and vagus were intact. Bronchoscopy and oesophagoscopy were normal. The patient required three units of blood during the operation and remained cardiovascularly stable, apart from one episode of bradycardia that responded to atropine. The external and internal jugular veins were ligated, haemostasis was secured, the strap muscles were repaired and the cervical fascia closed around the sternomastoid that was loosely approximated. A Yates drain was placed and the patient was admitted to the intensive-care unit. He remained intubated until day 6 , by day 9 he was able to swallow foods, and he was discharged home on day 10. At 6-week follow-up, there was some numbness distal to the skin wound, and slight pain and weakness on left sternomastoid movement. Following further physiotherapy the patient made a full recovery as indicated by review at 11 weeks.

\section{DISCUSSION}

Kickback occurs when the tip of a chainsaw suddenly engages with a solid object, causing the saw to fly up in the air. Safety features designed to minimize kickback include the tip guard and a chain brake that stops the forward motion of the chain within milliseconds. A safety chain with a sloping rather than square-shouldered raker and an asymmetrical or banana blade also reduces the incidence of kickback. ${ }^{5}$ In the case reported here, the tip guard was missing. There is no legislation in Queensland compelling the use of any of the above safety features, many of which are removed or modified in order to increase ease of handling of the saw.

The initial management of penetrating neck trauma follows standard trauma resuscitation principles, including immediate airway control with early endotracheal intubation, cricothyroidotomy or tracheostomy for actual or impending airway compromise. Clinical evidence of pneumothorax or haemothorax is treated by intercostal drainage. Venous access with two large-bore intravenous cannulae is established and external haemorrhage tamponaded by direct pressure, without the use of clamping. The wound is gently examined in order to determine the zone of penetration and to establish whether the platysma has been breached, by careful spreading of the skin if necessary, but without blind probing. Assessment is made for evidence of vascular, airway and respiratory, neurological, oesophageal, skeletal and soft tissue injuries that may be seen in descending order of frequency. ${ }^{2}$ During resuscitation, the patient is kept supine or in slight Trendelenburg position to reduce the risk of air embolism. A chest radiograph is performed in order to exclude pneumothorax, haemothorax, pneumomediastinum and subcutaneous emphysema. Stable patients may also require a cervical spine radiograph, provided close observation is maintained throughout. All patients receive tetanus and antibiotic prophylaxis. Definitive care includes immediate surgery for unstable patients or those presenting with clinical findings that suggest significant injury (see Table 1). 
Chainsaw neck injury

Table 1. Absolute indications for exploratory surgery in penetrating neck trauma

Vascular

Persistent shock and unstable vital signs

Extensive blood loss or continued bleeding

Other signs of vascular injury: expanding haematoma, absent or diminished pulse, bruit, haemothorax.

Airway and respiratory tract

Air leak from wound, airway obstruction, respiratory distress

Hoarseness, dysphonia, haemoptysis, crepitation, subcutaneous emphysema

Neurological

Peripheral deficit of cranial or sympathetic nerves, brachial plexus and spinal cord

Evolving stroke

Oesophageal

Haematemesis, dysphagia

Mechanism

Gunshot, shotgun wounds

Based on Caducci, ${ }^{2}$ and Mansour ${ }^{9}$

Note that in stable patients, angiography may be performed preoperatively, particularly in Zone I and Zone III injuries.

All other patients with actual penetration of the platysma are admitted. There is controversy as to whether stable patients without positive findings should then undergo mandatory surgery or be carefully observed, with surgery performed selectively following arteriography, panendoscopy and oesophagrams as indicated.

Those who advocate mandatory exploration argue that the initial history and clinical examination alone are not sensitive indicators of significant injury, and that delayed management of vascular and oesophageal injuries increases morbidity and mortality. ${ }^{6}$ Furthermore, significant injuries may still be missed even with four-vessel arteriography, panendoscopy and oesophagrams, and significant morbidity is rarely attributable to a negative neck exploration. ${ }^{7}$

Authors who favour selective management consider the 40-60\% negative exploration rate with mandatory surgery unacceptable. They point out that significant lesions are also missed during surgical exploration. ${ }^{8}$ In addition, a non-exploratory work-up including angiography, panendoscopy and oesophagrams is sufficiently sensitive that increased morbidity and mortality do not result, ${ }^{9}$ and the negative exploratory rate is significantly lower at $12-22 \% .^{7}$ Finally, the average length of hospital stay of patients managed selectively who do not require surgery ( 2.8 days) is shorter than the average length of stay of patients undergoing mandatory surgery and a negative exploration (4.2 days), with similar overall costs. ${ }^{10}$

Decisions regarding selective management assume that experienced radiologists, endoscopists and angiographers are available on a 24-h basis, with suitable medical and nursing staff available to allow frequent repeated examination. This approach would therefore only be appropriate in large teaching hospitals. Otherwise, mandatory exploration for penetrating neck trauma, particularly in Zone II injuries, with their ease of exploration and minimal operative complications, would seem to be more suitable for all other institutions caring for trauma, and for those surgeons who only rarely deal with this type of injury.

\section{REFERENCES}

1. Wood J., Fabian T.C. \& Mangiante E.C. (1989) Penetrating neck injuries: recommendations for selective management. Journal of Trauma 29, 602605.

2. Carducci B., Lowe R.A. \& Dalsey W. (1986) Penetrating neck trauma: consensus and controversies. Annals of Emergency Medicine 15, 208-215.

3. Thal E.R. \& Meyer D.M. (1992) Penetrating neck trauma. Current Problems in Surgery 29, 13-14.

4. Ordog G.J. (1987) Penetrating neck trauma. Journal of Trauma 27, 543-553.

5. Pratt L.W. (1985) Chainsaw injuries of the head and neck. Ear Nose and Throat Journal 64, 215-222. 
6. Bishara R.A., Pasch A.R., Douglas D.D., Schuler J.J., Lim L.T. \& Flanigan D.P. (1986) The necessity of mandatory exploration of penetrating Zone II neck injuries. Surgery 100, 655-658.

7. Rathlev N.K. (1990) Penetrating neck trauma: mandatory versus selective operation. Journal of Emergency Medicine 8, 75-78.

8. Obeid F.N., Haddad G.S., Horst H.M. \& Bivins B.A. (1985) A critical reappraisal of a mandatory exploration policy for penetrating wounds of the neck. Surgery, Gynecology and Obstetrics 160, 517522.

9. Mansour M.A., Moore E.E., Moore F.A. \& Whitehill T.A. (1991) Validating the selective management of penetrating neck wounds. American Journal of Surgery 162, 517-520.

10. Noyes L.D., McSwain N.E. \& Markowitz I.P. (1986) Panendoscopy with arteriography versus mandatory exploration of penetrating wounds of the neck. Annals of Surgery 204, 21-31. 